\title{
GeoBoids: A Mobile AR Application for Exergaming
}

\author{
Robert W. Lindeman ${ }^{1,2_{\star}}$, Gun Lee ${ }^{1}$, Leigh Beattie ${ }^{1}$, Hannes Gamper ${ }^{1}$, Rahul Pathinarupothi ${ }^{1}$, Aswin Akhilesh ${ }^{1}$ \\ The Human Interface Technology Laboratory New Zealand, University of Canterbury ${ }^{1}$ \\ Human Interaction in Virtual Environments Lab, Worcester Polytechnic Institute ${ }^{2}$
}

\begin{abstract}
We have designed a mobile Augmented Reality (AR) game which incorporates video see-through and spatialized audio AR techniques and encourages player movement in the real world. In the game, called GeoBoids, the player is surrounded by flocks of virtual creatures that are visible and audible through mobile AR application. The goal is for the player to run to the location of a GeoBoid swarm in the real world, capture all the creatures there, then run to the next swarm and repeat, before time runs out, encouraging the player to exercise during game play. The most novel elements of the game are the use of audio input and output for interacting with the creatures. The interface design of the game includes AR visualization, spatialized audio, touch gestures and whistle interaction. Feedback from users in a preliminary user study was mostly positive on overall game play and the design of the UI, while the results also revealed improvements were needed for whistle interaction and the visual design of the GeoBoids.
\end{abstract}

Keywords: Augmented Reality, Exergaming, Spatialized Audio, Mobile, Gaming.

Index Terms: H.5.1 [Information Interfaces and Presentation]: Multimedia Information Systems - Artificial, augmented, and virtual realities

\section{INTRODUCTION}

Mobile Augmented Reality (AR) platforms support many possible applications, including information display for annotation [1], self-guided tours $[2,5]$, visualization for city planning [3], and entertainment $[4,5]$. New hardware initiatives, such as Epson's Moverio [6] or Google's Project Glass [7], provide a glimpse of what light-weight, wearable, always-available display technology might look like. Such devices provide see-through visual displays, wireless Internet connectivity, location and motion sensors, and audio interfaces. We are interested in exploring the design space of applications for devices with these capabilities, focusing here on entertainment.

\section{AR FOR ENTERTAINMENT}

Several innovative mobile AR games have been designed for use in an outdoor setting. The iOS game Star Wars Arcade: Falcon Gunner [8] puts the player in the gunner turret of the Millennium Falcon, with a mode that blends graphical elements with the view captured from the mobile device camera, and another mode that is purely virtual. Paranormal Activity: Sanctuary [9] is an actionhorror game where the player has to rid their local area of evil ghosts. It combines a map mode, a spell-casting mode where the

\footnotetext{
* email address: gogo@wpi.edu
}

camera captures stars sketched by the player, and a first-person view where 3D-registered virtual ghosts are displayed in physical locations, such as cemeteries and churches. Ghosts can be captured by snapping a picture of them. AR! Pirates [10] is a game that uses the camera to capture a simple AR marker (three dots), and embeds a pirate ship siege scene into the view. The player can shoot the ships using weapons. Lacking from most mobile AR games is any use of audio as input, as well as a design that encourages physical player movement over large distances.

\section{GeoBoids: Creatures from the Eighth Dimension}

GeoBoids is a game designed to combine fast-paced, arcade-style action with large-scale physical user movement for exergaming. The concept is that geometric creatures from the eighth dimension are visible in our dimension using a specially equipped device (the player's smartphone). They congregate in flocks in certain areas of our world. The player's goal is to travel to those places identified on a map, and collect GeoBoids, in order to help them return to their own dimension. Each species of GeoBoid has its own properties, including color, shape, flying pattern, active time of day/night, and affinities (Figure 1). Two main game-play modes have been implemented: Field Mode and Arcade Mode. Other modes, including breeding and trading GeoBoids through social networking sites, are being explored.

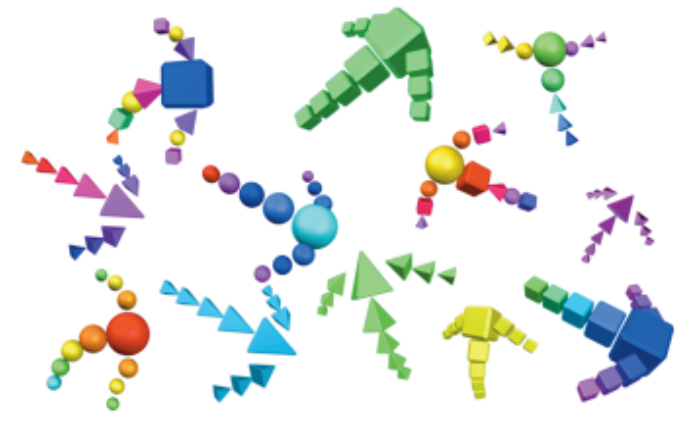

Figure 1: Visual concept of GeoBoids.

\subsection{Exergaming in Field Mode}

Field Mode play assumes the player is standing in a large open space, about the size of a football field. In this mode, the player sees a digital map of the local area, with locations of GeoBoid flocks displayed as overlays (Figure 2a). These locations are randomly generated each time the player plays a level in Field Mode. The player position is denoted by an oriented arrow, which is updated dynamically as the player moves around the physical world. An audible sonar "ping" is played using spatialized audio according to the distance and direction toward a GeoBoid flock, helping the player to find the nearest GeoBoid. Once the player is within range of a flock, play switches to Arcade Mode. 


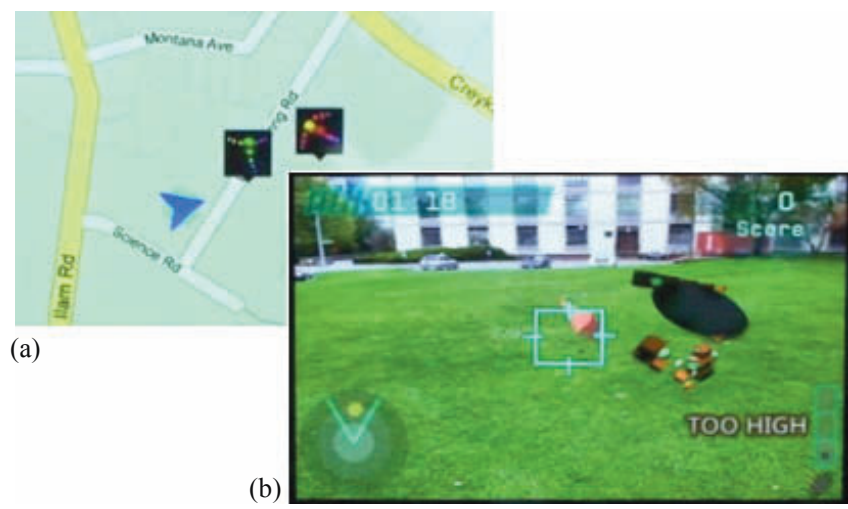

Figure 2: (a) Field Mode Map; (b) Arcade Mode View

\subsection{Arcade Play in AR View}

In Arcade Mode, the player is faced with waves of GeoBoids with varying attributes, and must capture all GeoBoids in the level within a set amount of time to move on to the next level. Capturing a GeoBoid results in points and added time for the player, and different GeoBoids have different point and time values, depending on the difficulty of capture (Figure $2 b$ ). The flocking behavior of the GeoBoids is implemented using Craig Reynolds' OpenSteer code [11], which we ported to the Google Android platform, and spatialized audio is supported through FMOD [12] which has also been ported to Android.

The game uses video see-through AR using tracking information from motion and location sensors. As a result, the GeoBoids appear visually and aurally as if they are situated in the real world. Interestingly, though no image-based registration is done, feedback from user testing showed that players thought the GeoBoids were interacting with the environment.

The motion sensors are used to allow "point-and-shoot" interaction, with a swipe gesture used to capture the GeoBoid within the on-screen reticle. For the GeoBoid in the reticle, a health meter is displayed on the left side of the reticle, allowing the player to maintain focus, while still receiving game status. A continuously updating radar shows the location of GeoBoids around the player, and an on-screen countdown timer is used to provide motivation for the player to capture all the GeoBoids as quickly as possible. We have implemented several game levels with increasing difficulty as the player progresses.

One of the unique game mechanics is the use of whistle as an input option (Figure 2). Within the AR view, if the player whistles into the phone at the correct pitch and for the correct duration, the GeoBoids get scared. As a result, they flock more tightly, thereby making them easier to capture. Interactive feedback using an onscreen audio level is given to the player to let them know if they are whistling correctly.

\section{User Feedback}

The GeoBoids game was demonstrated to the general public (about 40-50 people) during an Open House at the HIT Lab NZ. The feedback was positive and the overall game experience appeared to be compelling. Also, semi-structured user testing was conducted with students at the lab. Because of time and space limitations, only Arcade Mode was evaluated. Eight students participated, were asked to play the game freely for 10-20 minutes and gave feedback through a questionnaire, which included questions on a five-point scale, 1: fully disagree and 5: fully agree, as well as those asking for free-form text about features they liked and improvements needed. Table 1 summarizes the results of the questionnaire rating questions.
Table 1: User Feedback (1: fully disagree - 5: fully agree)

\begin{tabular}{l|c}
\hline \multicolumn{1}{c|}{ Question } & $\begin{array}{c}\text { Responses } \\
\text { (Mean } / \text { S.d. }\end{array}$ \\
\hline I learned how to play the game pretty quickly. & $4.75 / 0.463$ \\
Capturing the GeoBoids was pretty easy. & $4.38 / 0.916$ \\
The "swipe" gesture for capturing worked well for me. & $4.13 / 1.126$ \\
The radar display was helpful for doing well. & $4.13 / 1.356$ \\
It was clear to me what the radar display was showing. & $4.00 / 1.195$ \\
The graphical elements were attractive. & $4.13 / 0.641$ \\
Using the GUI, it was easy to keep track of things. & $3.81 / 0.923$ \\
The "whistle" input was helpful for doing well. & $2.38 / 1.188$ \\
The "whistle" input was fun. & $2.63 / 1.061$ \\
It was clear what the "whistle" input was used for. & $3.13 / 1.246$ \\
The whistle meter made it easy to understand how I had & $2.50 / 0.756$ \\
to whistle. & \\
Overall, I thought this game was fun. & $4.13 / 0.641$ \\
\hline
\end{tabular}

The participants most liked that the game was integrated with the real world, providing an AR-style game play environment, the active movement involved in the game play, and that it included new types of interaction such as whistle detection.

When asked the features that they least liked or which needed improvement, the participants mentioned that whistling was not working well and made them tired, the visual appearance of GeoBoids was not that attractive, and that the GeoBoids were less actively engaging with the player (such as attacking the player). Some participants appeared to not be able to whistle at all, suggesting other types of input should be considered. Blowing and shouting were suggested as alternative forms of audio input.

\section{Conclusion}

We have designed a mobile AR game called GeoBoids in which the player is presented with flocks of virtual creatures that are visible and hearable using a mobile device. The game interface design elements included AR visualization, spatialized audio presentation, touch gesture interaction, and whistle recognition.

Based on the user feedback from a preliminary user study, we are planning to improve whistle recognition to work in more general cases, and improve the design of visual elements. In the future, we plan to investigate motion gestures as another modality of user interaction. Finally, the exergaming aspects of the game will be evaluated and refined.

\section{REFERENCES}

[1] Wither, J., DiVerdi, S., Höllerer, T. (2009). Annotation in outdoor augmented reality. Computers \& Graphics, 33(6), pp. 679-689.

[2] Feiner, S., MacIntyre, B., Höllerer, T., and Webster, T. (1997). A touring machine: Prototyping 3D mobile augmented reality systems for exploring the urban environment. Proc. ISWC'97 (First IEEE Int. Symp. on Wearable Computers), Oct. 13-14, 1997.

[3] Kato, H., Tachibana, K., Tanabe, M., Nakajima, T., Fukuda, Y. (2003). A city-planning system based on augmented reality with a tangible interface. Proc. ISMAR 2003, pp. 340-341.

[4] Starner, T., Leibe, B., Singletary, B., Pair, J. (2000). MINDWARPING: towards creating a compelling collaborative augmented reality game. Proc. IUI'00, pp. 256-259.

[5] Herbst, I., Braun, A.-K., McCall, R., Broll, W. (2008). TimeWarp: Interactive Time Travel with a Mobile Mixed Reality Game, Proc. MobileHCI 2008, pp. 235-244.

[6] http://www.epson.com/cgi-bin/Store/jsp/Product.do?sku=V11H423020

[7] http://en.wikipedia.org/wiki/Project_Glass

[8] http://www.thq.com/us/star-wars-flight-of-the-falcon/wireless

[9] http://paranormalactivitythegame.com/

[10] http://www.arpirates.com/

[11] http://opensteer.sourceforge.net/

[12] http://www.fmod.org/ 